\title{
COMPARACIÓN DEL ESTADO NUTRICIO EN ESCOLARES DE PLANTELES EDUCATIVOS CON Y SIN SERVICIO DE COMEDOR
}

Velia Herminia Castillo Pérez. ${ }^{1}$, Selene Agrimar Valverde Benavides ${ }^{2}$

Resumen. En este artículo se presenta el estudio efectuado para evaluar el estado nutricio de escolares, entre nueve y once años; pertenecientes, unos, a una escuela primaria con comedor y otros, a una escuela sin servicio de comedor escolar.

Mediante un muestreo no probabilístico, por conveniencia, se obtuvo una muestra de 29 niños para la escuela con comedor escolar y otra de 49 niños para la escuela sin servicio de comedor, en un rango de nueve a 11 años. Se les realizaron mediciones antropométricas y se definió el estado nutricional, mediante los indicadores de crecimiento según las tablas de la Organización Mundial de la Salud (OMS) del 2008. También se aplicaron cuestionarios para evaluar hábitos alimentarios y de estilo de vida en los niños que fueron evaluados antropométricamente, el cual constó de 30 ítems. Así mismo, se analizaron los menús que se les ofrecen a los niños de la escuela con comedor para observar el tipo y calidad de la dieta que se les brindaba. Con los resultados obtenidos se concluyó que el estado nutricional de los escolares de la escuela primaria con comedor es diferente en comparación con los escolares de la escuela primaria sin comedor, sin embargo en ambos planteles educativos se observan casos de malnutrición, por lo que no se puede afirmar que el contar o no con el servicio de comedor escolar tuviera influencia en el desarrollo de problemas nutricionales.

Palabras clave: Estado nutricio, comedor escolar, menú, obesidad, desnutrición.

\footnotetext{
${ }^{1}$ Dra. En Ciencias Administrativas Velia Herminia Castillo Pérez es Profesora adscrita de la Maestría en Administración de la Universidad Autónoma de Chihuahua, Cd. Juárez, Chih., México dcastillop@uach.mx y al Instituto Tecnológico de Ciudad Juárez, Cd. Juárez, Chih., México.

${ }^{2}$ Selene Agrimar Valverde Benavides es Licenciada en Nutriología, maestrante de Administración de la Universidad Autónoma de Chihuahua, México. licvalverde.sel@gmail.com
} 


\section{Introducción}

A lo largo de la vida, la nutrición, es uno de los elementos clave para la Salud, el desempeño físico y mental y la productividad de los individuos. La desnutrición en el niño es el resultado de una dieta inadecuada: insuficientes alimentos nutritivos, en cantidad y/o calidad; el efecto acumulativo de episodios repetidos de enfermedades infecciosas o de otros padecimientos; servicios de salud deficientes; saneamiento ambiental inadecuado y prácticas inapropiadas de cuidado en el hogar (ENSANUT, 2006).

La obesidad es el resultado de un desequilibrio entre la ingestión y el gasto energético. Este desequilibrio es frecuentemente consecuencia de la ingestión de dietas con alta densidad energética y bajas en fibra; y de bebidas azucaradas, en combinación con una escasa actividad física. Esta última se ha asociado a la urbanización, al crecimiento económico y a los cambios en la tecnología para la producción de bienes y servicios, así como a los estilos de vida y de recreación.

\section{Objetivo del Estudio}

Evaluar el estado nutricio de escolares, entre nueve y 11 años, pertenecientes, unos a una escuela primaria con comedor $y$, otros a una escuela sin servicio de comedor.

\section{Marco Teórico}

En México, entre 1999 y 2006, la prevalencia combinada de sobrepeso y obesidad en niños y niñas en etapa escolar aumentó un tercio y en 2012 la reducción es menor a un punto porcentual (ENSANUT, 2012). Los resultados señalan la urgencia de aplicar medidas conducentes a la prevención de obesidad en los escolares (ENSANUT, 2006).

Según información recabada por la Encuesta Nacional de Salud y Nutrición llevada a cabo en el año 2006, en México, se muestra que la prevalencia de desnutrición en escolares de 
cinco a once años ha disminuido en comparación con los datos obtenidos por esta misma encuesta en 1999 (Payán, 2006). La Encuesta Nacional de Salud del 2012 en México, muestra la prevalencia de sobrepeso y obesidad en niños, entre cinco y once años, con tendencia a la baja de 37.4 a 36.9 y en las niñas de 32.3 a $32 \%$ con lo que se refuerza la necesidad de tomar acciones que reviertan el fenómeno con mayor rapidez.

Aranceta (2008) menciona que los comedores escolares cumplen con una función muy importante de carácter nutricional y educativo, contribuyen a la adquisición de hábitos alimenticios, además de fomentar la socialización. Muchos de los menús ofertados en este tipo de servicio no van de acuerdo a las guías alimenticias para este colectivo. Un error muy notorio es el aporte deficiente de verduras, frutas y carnes blancas, además de la saturación del uso de grasas para los procesos culinarios de los alimentos, por lo que es de gran necesidad que se incluyan recomendaciones nutricionales para estas comidas escolares, incluyendo información de guías alimenticias, tamaño de raciones, atención a necesidades especiales, así como medidas de higiene en la manipulación y preparado de los alimentos (Aranceta et al., 2008).

De acuerdo a cifras de la Organización de las Naciones Unidas para la Alimentación y la Agricultura (FAO por sus siglas en inglés) se ha registrado una polarización en los problemas nutricionales de México, que consiste en la persistencia de altas tasas de desnutrición en el sur del territorio y en las zonas rurales, así como el aumento de las tasas de sobrepeso y obesidad en el norte y en zonas urbanas (García, 2010).

En México, desde 1929, se aplican programas de ayuda alimentaria por parte del gobierno a grupos vulnerables; se realizan mediante el Sistema Nacional para el Desarrollo Integral de la Familia (DIF), a través del Programa de Raciones Alimentarias (Desayunos EscolaresDIF). Estos programas son los únicos que han sobrevivido a los cambios sexenales, sin embargo, carecen de evaluaciones sistemáticas que permitan atribuir un cambio o beneficio debido a su implantación. López et al (2005), entre los años 2002 y 2003, practicaron un 
estudio en 17 municipios del estado de Sonora, México, con el objetivo de determinar el efecto de un programa de desayunos escolares sobre la prevalencia de obesidad y factores de riesgo cardiovasculares en niños sonorenses. Fue un estudio longitudinal en 254 niños del Programa de Desayunos Escolares (PDE), la evaluación se llevó a cabo al inicio y al final del ciclo escolar (nueve-meses) y fueron comparados con un grupo control ( $\sin$ PDE, $n=106$ ). Se utilizó el índice de masa corporal para la edad (IMC/edad) y se midió la composición corporal por bioimpedancia eléctrica. En una submuestra de 264 niños (PDE y controles) se determinó colesterol total, triglicéridos y glucosa, en ayuno. Los resultados indicaron que el IMC en niños del PDE y sus controles no mostró diferencia al inicio y final del ciclo escolar. Asimismo, el porcentaje de sobrepeso y obesidad no se modificó al final del Programa y el porcentaje de grasa corporal no mostró cambios. En conclusión, no se encontró evidencia de un efecto negativo del PDE sobre los factores de riesgo para obesidad y riesgo de enfermedad cardiovascular en niños del estado de Sonora (López et al., 2005).

Aranceta et al (2008) presenta un estudio realizado en algunos comedores escolares del municipio de Madrid en 2006; donde ponía en evidencia que más del 28\% del aporte calórico diario procedía de la ingesta realizada en el comedor escolar. El análisis de los menús ofertados en estos centros evidenciaba la presencia elevada de carnes y, por el contrario, la oferta muy limitada de preparaciones de pescado. La oferta de verduras se consideraba aceptable, pero la de legumbres, frutas y cereales era inferior a lo deseable. Por el contrario, la ingesta de dulces, carnes y embutidos era elevada (Aranceta et al., 2008).

El lunes 23 de agosto de 2010, en México, D.F., se publicó en el Diario Oficial de la Federación, el Acuerdo Nacional para la Salud Alimentaria - Estrategia contra el Sobrepeso y la Obesidad - Programa de Acción en el Contexto Escolar.

Acuerdo mediante el cual se establecen los lineamientos generales para el expendio o distribución de alimentos y bebidas de consumo escolar de los planteles de educación básica. El pasado $1^{\circ}$ de enero de 2011 entraron en vigor ciertos lineamientos para las escuelas 
públicas y particulares del Sistema Educativo Nacional en los establecimientos de consumo escolar; estas disposiciones tienen, entre otros, los siguientes objetivos: Promover que en los establecimientos de consumo escolar se preparen y expendan alimentos y bebidas que promuevan una alimentación correcta; encaminar los esfuerzos y acciones para que en las entidades federativas se regule, de manera coordinada y unificada, la operación de este tipo de establecimientos a fin de constituir normas claras sobre el tipo de productos que se recomienda elaborar, expender o distribuir en las escuelas de Educación Básica y las medidas de higiene que deberán acatarse para hacer de éstas espacios saludables (Comedores Industriales, 2011).

\section{La etapa escolar}

A la edad escolar también se le conoce como infancia media, la cual describe a los niños de entre cinco y 10 años de edad. A las niñas de nueve a 11 años y a los niños de 10 a 12 años se les conoce como preadolescentes, término que también puede emplearse para describir la edad escolar (Brown, 2006).

En estas edades, el incremento en el peso y la estatura son constantes y conforme aumenta la edad, éstos son mayores en mujeres que en hombres. A los seis años no existen diferencias de peso y estatura entre niños y niñas; es hasta los 10 años cuando se comienzan a notar estas diferencias (Casanueva et al., 2008).

\section{Conductas alimenticias}

Los padres y hermanos mayores ejercen una enorme influencia en la actitud del niño hacia la comida. Las preferencias de los padres en los alimentos, así como sus prácticas culturales, afectan los gustos y aversiones en los niños. Los padres son responsables del ambiente alimenticio de la casa, de la elección del tipo de alimento y de cuándo se sirven, y el niño es el que elige cuánto es lo que come. Por lo que resulta muy importante que los padres sean 
modelos positivos para sus hijos al mostrarles conductas alimenticias saludables y que le proporcionen al niño la guía necesaria para que él tenga la posibilidad de elegir alimentos saludables cuando esté fuera de casa (Brown, 2006).

Medidas correctoras y de prevención para la obesidad y desnutrición en escolares

La Estrategia Mundial de la OMS sobre Régimen Alimentario, Actividad Física y Salud, adoptada por la Asamblea de la Salud en 2004, describe las acciones para apoyar la adopción de dietas saludables y una actividad física regular. Dicha estrategia pide a todas las partes interesadas que actúen a nivel mundial, regional y local, y tiene por objetivo lograr la reducción significativa de la prevalencia de enfermedades crónicas y de sus factores de riesgo, así como de las dietas poco saludables y de la inactividad física. El Departamento de Nutrición para la Salud y el Desarrollo, complementa la labor de la OMS con objetivos estratégicos como el fomento del consumo de dietas saludables y mejoría en el estado nutricional de la población a lo largo de toda la vida, especialmente entre los más vulnerables, “para lo cual proporciona apoyo a los países para que elaboren y apliquen programas y políticas nacionales intersectoriales de alimentación y nutrición que permitan hacer frente a la doble carga de enfermedades relacionadas con la nutrición y contribuir a la consecución de los Objetivos de Desarrollo del Milenio" (OMS, 2006).

\section{Comedores escolares}

Los comedores escolares influyen muchísimo en la salud de los niños ya que allí realizan la comida más importante del día y aprenden hábitos alimentarios. Algunos objetivos y funciones más comunes de los comedores escolares son: proporcionar el desayuno a aquellos alumnos que por diferentes motivos no lo realizan en sus casas, enseñar o potenciar buenos hábitos a la hora de comer (lavarse las manos antes, comer utilizando los diferentes cubiertos 
correctamente, aprender a compartir ese espacio con otras personas respetuosamente, disfrutar de una dieta lo más variada posible, etc.), potenciar el compañerismo, enseñar a aprender a comer lo que haya y no sólo lo que les gusta (Arnau, 2008).

En México, el programa de desayunos escolares del DIF nacional, a sus casi 80 años del primer plan alimentario proporcionado por esta institución, decidió cambiar sus objetivos. Durante décadas, se dedicó a alimentar a los niños del país, sin tomar en cuenta los cambios generacionales, hasta que se dio cuenta que gran parte de los niños de ayer dejaron de ser desnutridos y hoy la mayoría son mal nutridos, obesos, que desayunan en exceso o simplemente salen de sus casas rumbo a la escuela sin haber probado alimentos, no porque no tengan, sino por el acelerado ritmo de vida de sus padres. Por eso, se decidió modificar el famoso desayuno de la cajita de leche, la palanqueta y la galleta de chocolate con vainilla, por leche descremada, galleta con fibra y fruta. La recomendación ideal es hacer un desayuno, una comida, dos colaciones y la cena. Con un desayuno no se abatirá ni la desnutrición, ni se combatirá el sobrepeso y la obesidad, pero se da una aportación pequeña para atender esta gran problemática (Rodríguez, 2008).

\section{Metodología}

Por medio de un muestreo no probabilístico, por conveniencia, se obtuvo una muestra de 29 niños para la escuela con comedor escolar y otra de 49 niños para la escuela sin servicio de comedor, en un rango de edad entre nueve a 11 años. Se realizaron mediciones antropométricas: peso y talla, y, se definió el estado nutricional, de cada niño, mediante los indicadores de crecimiento según las tablas de la Organización Mundial de la Salud (OMS) del 2008. También se aplicó, a todos los niños de la muestra, un cuestionario de 30 ítems, para evaluar sus hábitos alimenticios y su estilo de vida. Así mismo, se analizaron los menús 
que se les ofrecían en la escuela con comedor; para observar el tipo y calidad de la dieta brindada.

Para la realización de este estudio se utilizaron los siguientes instrumentos y materiales: Báscula, estadímetro, hojas de máquina (test), lápices y plumas, computadora.

\section{Interpretación de indicadores de crecimiento}

Las líneas de referencia de las curvas de crecimiento se llaman líneas de puntuación z debido a que se basan en puntuación z, también conocidas como puntuación de desviación estándar (DE). Las puntuaciones z o puntuaciones de DE se usan para describir la distancia que hay entre una medición y la mediana (OMS, 2008).

\section{Análisis de resultados}

Se pasó toda la información recabada a una hoja de cálculo Excel 2007, para posteriormente obtener el análisis del estado nutricio de cada uno de los niños participantes, mediante el software EPI INFO 2007, versión 3.4.3, del cual se obtuvieron los percentiles y puntuaciones $\mathrm{Z}$ del peso para la edad, talla para la edad e IMC de cada uno de los niños participantes. Luego se procedió a procesar toda la información tanto antropométrica como la del cuestionario con el uso del software analizador de datos estadísticos (STATA 2009, versión 11.1) y así se obtuvo de los variables categóricos porcentajes y de las continuas medidas de dispersión.

\section{Resultados}

Descripción de la muestra:

En la Tabla No.1 se muestra el total de niños de nueve a 11 años que participó en el estudio por escuela, siendo el 53\% alumnos de la Escuela Vicente Guerrero (con comedor escolar) y $46 \%$ de la Escuela América. 
TABLA No. 1. Niños de nueve a 11 años que participaron en el estudio.

\begin{tabular}{llll}
\hline ESCUELA & FRECUENCIA & \% & FREC. ACUM \\
\hline Vicente Guerrero (C) & 29 & 53.7 & 53.7 \\
América (NC) & 25 & 46.3 & 100 \\
TOTAL & 54 & 100 & \\
\hline
\end{tabular}

Fuente: Elaboración propia.

Del total de niños mencionados en la Tabla No. 1, el 41.4\% fueron niños y el $58.6 \%$ niñas de la Escuela Vicente Guerrero, así como el 36\% niños y 64\% niñas de la Escuela América, como se muestra en la Gráfica No.1.

GRÁFICA No.1 Niños que participaron en el estudio.

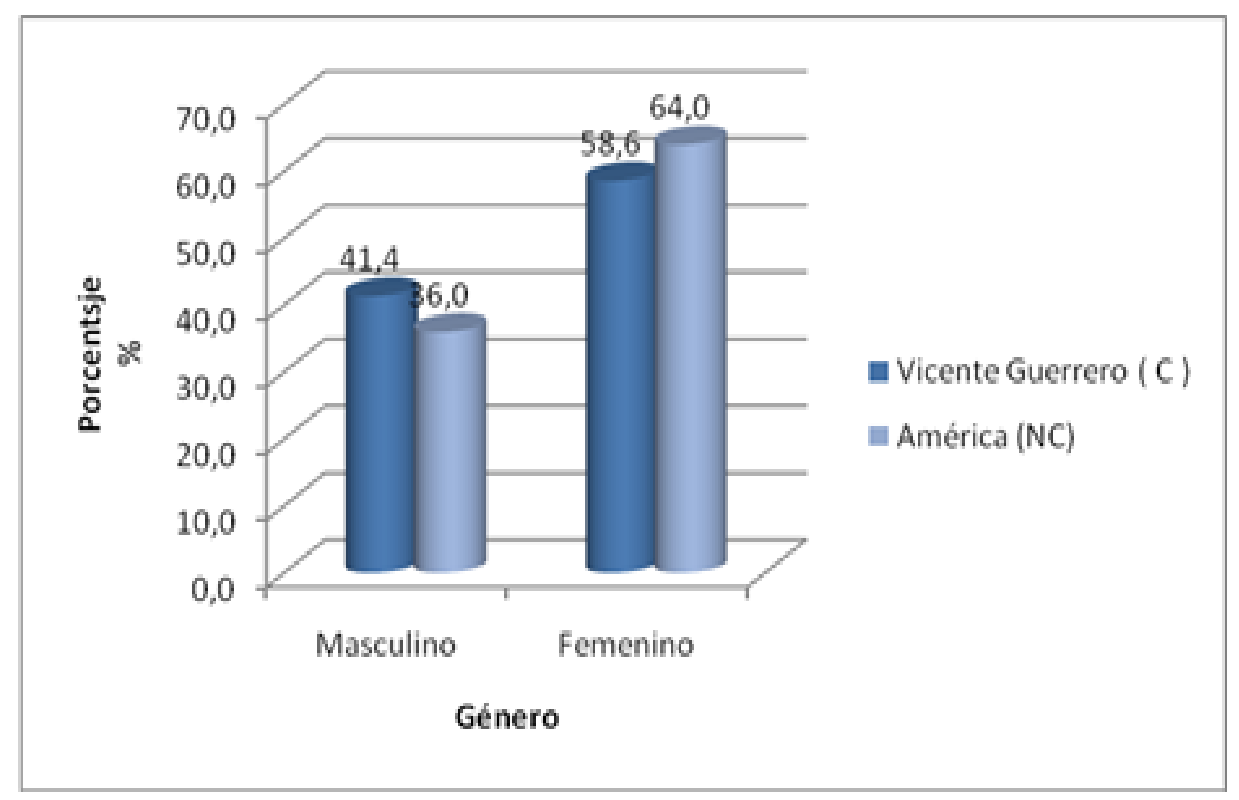

Fuente: Elaboración propia. 


\section{Antropometría:}

Clasificando a los alumnos por escuela, se encontró una media de peso de $35.9 \mathrm{~kg}$ para la escuela C y de 34.6 para la NC (Tabla No.2), como lo muestra la tabla, la media del peso corporal en kg del total de niños participantes, circula alrededor de los $35 \mathrm{~kg}$.

TABLA No. 2. Peso en kg de los participantes.

\begin{tabular}{llll}
\hline PESO KG & & & \\
\hline ESCUELA & MEDIA & $\begin{array}{l}\text { DESVIACIÓN } \\
\text { ESTÁNDAR }\end{array}$ & FRECUENCIA \\
Vicente Guerrero ( C ) & 35.9 & 7.2 & 29 \\
América (NC) & 34.6 & 8.3 & 25 \\
TOTAL & 35.3 & 7.7 & 54 \\
\hline
\end{tabular}

Fuente: Elaboración propia.

Respecto a la talla en cm, (Tabla No.3), se observa un media de $147.8 \mathrm{~cm}$ para la escuela Vicente Guerrero y de $143 \mathrm{~cm}$ para la escuela América.

TABLA No. 3. Talla en cm de los niños del estudio por escuela.

\begin{tabular}{|c|c|c|c|}
\hline \multicolumn{4}{|l|}{ TALLA CM } \\
\hline ESCUELA & MEDIA & $\begin{array}{l}\text { DESVIACION } \\
\text { ESTÁNDAR }\end{array}$ & FRECUENCIA \\
\hline $\begin{array}{l}\text { Vicente Guerrero } \\
\text { (C) }\end{array}$ & 147.8 & 5 & 29 \\
\hline América (NC) & 143 & 6.9 & 25 \\
\hline TOTAL & 145.6 & 6.4 & 54 \\
\hline
\end{tabular}

Fuente: Elaboración propia.

\section{Diagnóstico}

Para obtener el estado nutricio del escolar, se utilizaron tres índices de crecimiento (peso/edad, talla/edad e IMC) medidos en puntuación Z, obteniendo como resultado para el 
peso/edad una media de menos punto uno (-0.1) para ambas escuelas, siendo éste un valor normal con una desviación estándar de uno punto uno (1.1) para la escuela $\mathrm{C}$ y uno punto dos (1.2) para la NC (Tabla No.4).

TABLA No. 4. Puntuación $Z$ del índice peso para la edad.

\begin{tabular}{|c|c|c|c|c|c|}
\hline \multicolumn{6}{|l|}{$\mathbf{P} / \mathbf{E}_{-} \mathbf{Z}$} \\
\hline \multirow[b]{2}{*}{ ESCUELA } & \multicolumn{4}{|c|}{ EDAD AÑOS } & \\
\hline & 9 & 10 & 11 & TOTAL & \\
\hline \multirow{4}{*}{$\begin{array}{l}\text { Vicente } \\
\text { Guerrero ( C ) }\end{array}$} & 1.1 & -0.2 & -0.5 & -0.1 & MEDIA \\
\hline & 1 & 1 & 1 & 1.1 & DESVIACIÓN \\
\hline & & & & & ESTÁNDAR \\
\hline & 4 & 17 & 8 & 29 & FRECUENCIA \\
\hline \multirow[t]{4}{*}{ América (NC) } & -0.1 & -0.1 & 0.4 & -0.1 & MEDIA \\
\hline & 1.2 & 1.3 & 0 & 1.2 & DESVIACIÓN \\
\hline & & & & & ESTÁNDAR \\
\hline & 10 & 14 & 1 & 25 & FRECUENCIA \\
\hline \multirow[t]{4}{*}{ TOTAL } & 0.2 & -0.1 & -0.4 & -0.1 & MEDIA \\
\hline & 1.2 & 1.1 & 1 & 1.1 & DESVIACIÓN \\
\hline & & & & & ESTÁNDAR \\
\hline & 14 & 31 & 9 & 54 & FRECUENCIA \\
\hline
\end{tabular}

Fuente: Elaboración propia.

Mientras tanto, respecto a la talla para la edad, se obtuvo una media de punto nueve (0.9) para la primaria Vicente Guerrero y de punto cinco (0.5) para la América, ambos valores normales, con una desviación estándar de punto siete (0.7) y punto nueve (0.9) respectivamente. Tales resultados pueden apreciarse en la tabla No.5. 
TABLA No. 5. Puntuación $Z$ del índice talla para la edad.

\begin{tabular}{|c|c|c|c|c|c|}
\hline \multirow[b]{2}{*}{ ESCUELA } & \multicolumn{5}{|c|}{ EDAD AÑOS } \\
\hline & 9 & 10 & 11 & TOTAL & \\
\hline \multirow{3}{*}{$\begin{array}{l}\text { Vicente Guerrero ( } \\
\text { C ) }\end{array}$} & 1.3 & 0.8 & 0.8 & 0.9 & MEDIA \\
\hline & 0.6 & 0.7 & 0.8 & 0.7 & $\begin{array}{l}\text { DESVIACIÓN } \\
\text { ESTÁNDAR }\end{array}$ \\
\hline & 4 & 17 & 8 & 29 & FRECUENCIA \\
\hline \multirow[t]{3}{*}{ América (NC) } & 0.3 & 0.5 & 2.4 & 0.5 & MEDIA \\
\hline & 1 & 0.8 & 0 & 0.9 & $\begin{array}{l}\text { DESVIACIÓN } \\
\text { ESTÁNDAR }\end{array}$ \\
\hline & 10 & 14 & 1 & 25 & FRECUENCIA \\
\hline \multirow[t]{3}{*}{ TOTAL } & 0.6 & 0.7 & 0.9 & 0.7 & MEDIA \\
\hline & 1 & 0.8 & 0.9 & 0.8 & $\begin{array}{l}\text { DESVIACIÓN } \\
\text { ESTÁNDAR }\end{array}$ \\
\hline & 14 & 31 & 9 & 54 & FRECUENCIA \\
\hline
\end{tabular}

Fuente: Elaboración propia.

En cuanto al Índice de Masa Corporal (IMC), se presentó una media de menos cero punto ocho (-0.8) para el plantel C y de menos cero punto cinco (-0.5) para el plantel NC (Tabla no.6), siendo éste índice al igual que los anteriores, normal. 
TABLA No. 6. Puntuación Z del IMC según la edad.

\begin{tabular}{|c|c|c|c|c|c|}
\hline \multicolumn{6}{|l|}{ IMC_Z } \\
\hline \multirow{5}{*}{$\begin{array}{l}\text { ESCUELA } \\
\text { Vicente Guerrero ( } \\
\text { C) }\end{array}$} & \multicolumn{4}{|c|}{ EDAD AÑOS } & \\
\hline & 9 & 10 & 11 & TOTAL & \\
\hline & 0.7 & -0.1 & -1.4 & -0.8 & MEDIA \\
\hline & 1.3 & 1.7 & 1.7 & 1.7 & DESVIACIÓN \\
\hline & & & & & ESTÁNDAR \\
\hline & 4 & 17 & 8 & 29 & FRECUENCIA \\
\hline \multirow[t]{4}{*}{ América (NC) } & -0.4 & -0.5 & -1 & -0.5 & MEDIA \\
\hline & 1.4 & 1.5 & 0 & 1.4 & DESVIACIÓN \\
\hline & & & & & ESTANDAR \\
\hline & 10 & 14 & 1 & 25 & FRECUENCIA \\
\hline \multirow[t]{4}{*}{ TOTAL } & -0.1 & -0.7 & -1.3 & -0.6 & MEDIA \\
\hline & 1.4 & 1.6 & 1.6 & 1.6 & DESVIACIÖN \\
\hline & & & & & ESTÁNDAR \\
\hline & 14 & 31 & 9 & 54 & FRECUENCIA \\
\hline
\end{tabular}

Fuente: Elaboración propia.

Estado nutricio por plantel escolar según edad

En la Gráfica No.2, se muestra el porcentaje según estado nutricio que presentaron los escolares de la Primaria Vicente Guerrero, según su edad. Se pude apreciar como dato relevante, que el $10.3 \%$ presentó riesgo de sobre peso de los niños de nueve años, mientras que el alumno de 10 años presentó mayor porcentaje de emaciados en comparación a los de nueve y 11. 


\section{Gráfica No. 2. Alumnos de la Escuela con comedor por edad y estado nutricio.}

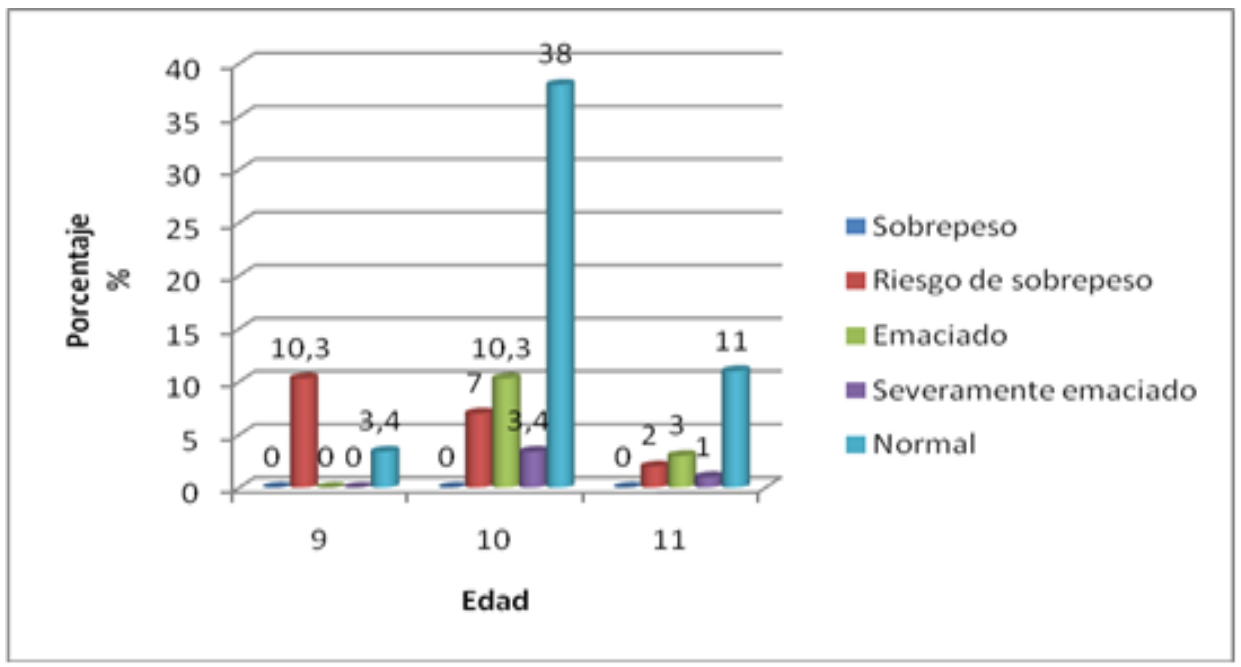

Fuente: Elaboración propia.

Respecto a la Escuela Primaria América (Ver gráfica No.3) el estado nutricio normal fue el que mayormente predominó, siendo más marcado en los niños de 10 años de edad, dato mencionado anteriormente, de riesgo de sobre peso con un ocho por ciento (8\%) para los niños de nueve y 10 años, encontrándose en estos últimos u porcentaje similar de emaciados.

Gráfica No. 3. Alumnos de la Escuela sin comedor por edad y estado nutricio.

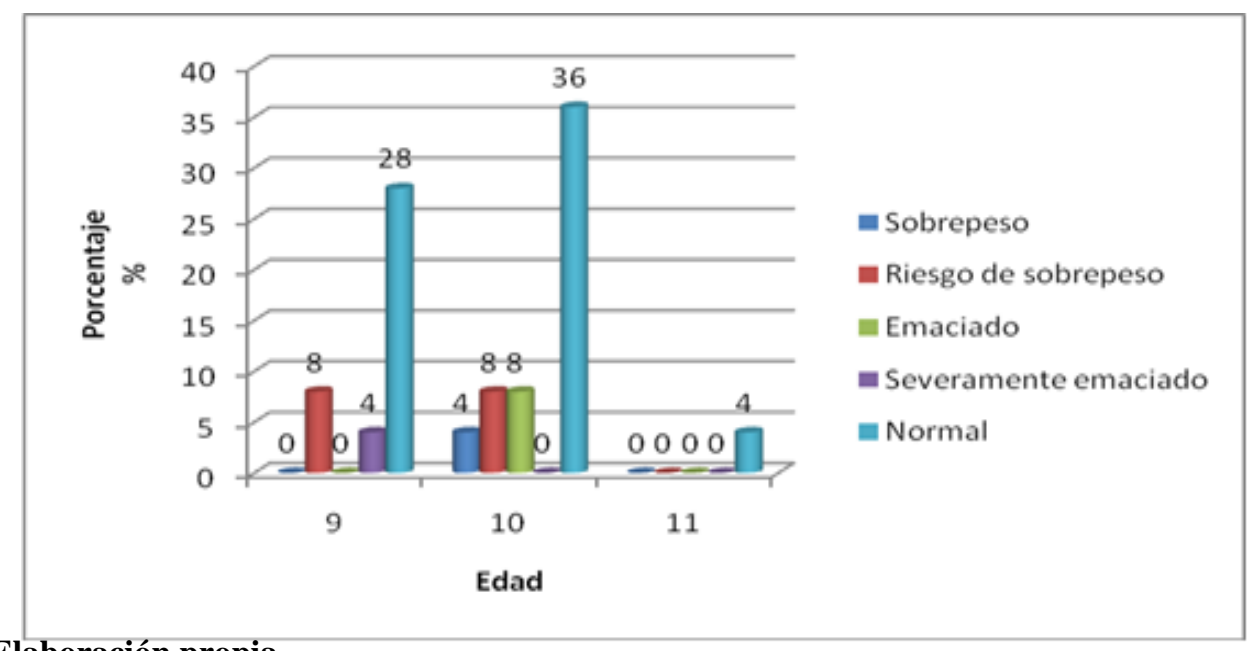

Fuente: Elaboración propia. 


\section{Comparación del estado nutricio por plantel}

Analizando la información por plantel, se observa mayor porcentaje de niños en condiciones normales en la escuela América, pero no teniendo ventaja tan significativa comparándola a la Vicente Guerrero, sin embargo esta última escuela presenta mayor porcentaje de riesgo de sobre peso, emaciación y emaciación severa en comparación al otro plantel educativo (Ver Gráfica No. 4).

Gráfica No. 4. Alumnos de las escuelas de acuerdo al estado nutricio.

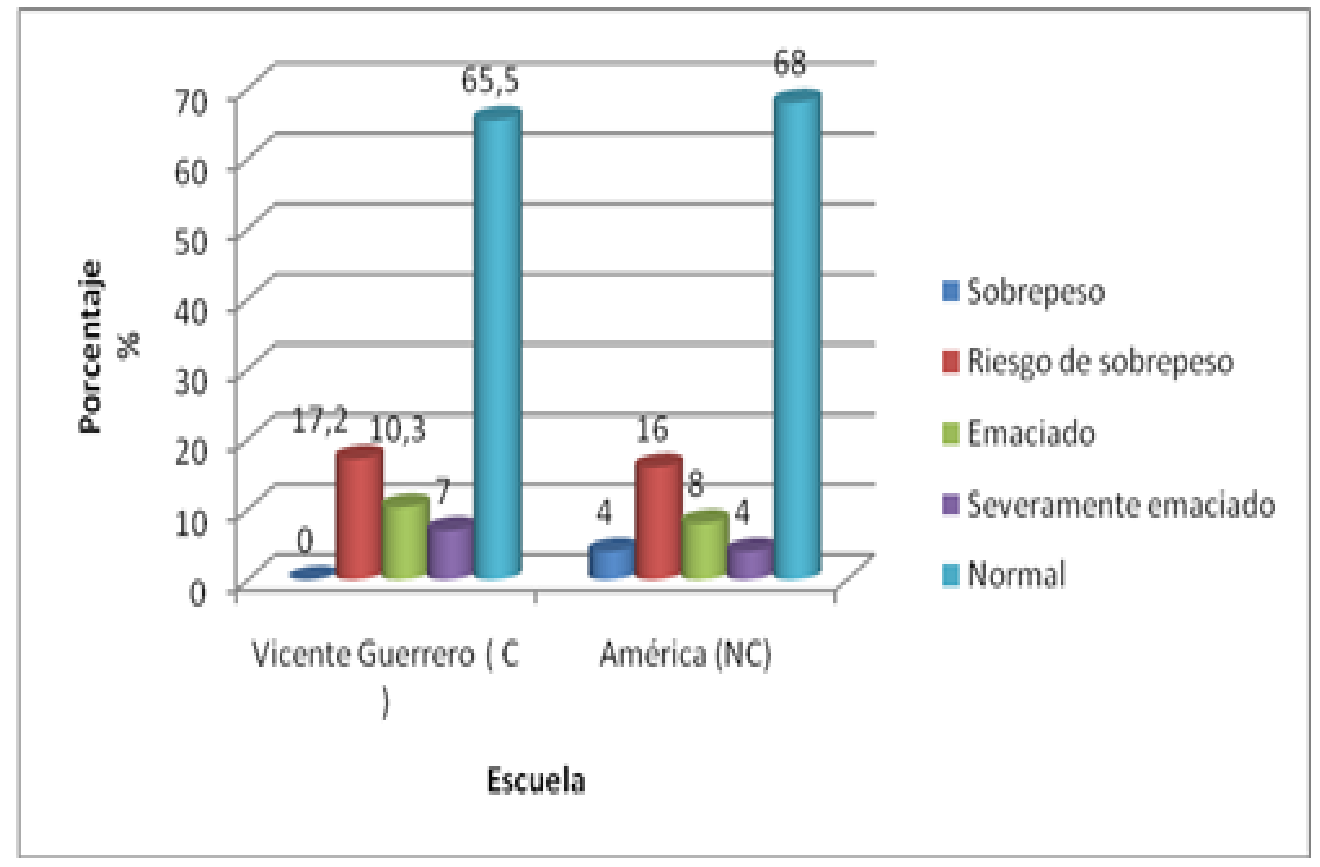

Fuente: Elaboración propia. 
Hábitos alimenticios y de estilo de vida

Posteriormente al análisis de la información antropométrica, se analizó el cuestionario aplicado a los escolares participantes, donde según muestra la Gráfica No.5, en ambas escuelas, los niños acostumbran dar cuatro comidas al día, sin embargo el tres punto cuatro por ciento $(3.4 \%)$, perteneciente a la Escuela $\mathrm{C}$ realiza dos comidas al día y el ocho por ciento $(8 \%)$ de la escuela NC realiza más de seis comidas al día.

\section{Gráfica No. 5. Número de comidas que realizan al día los alumnos.}

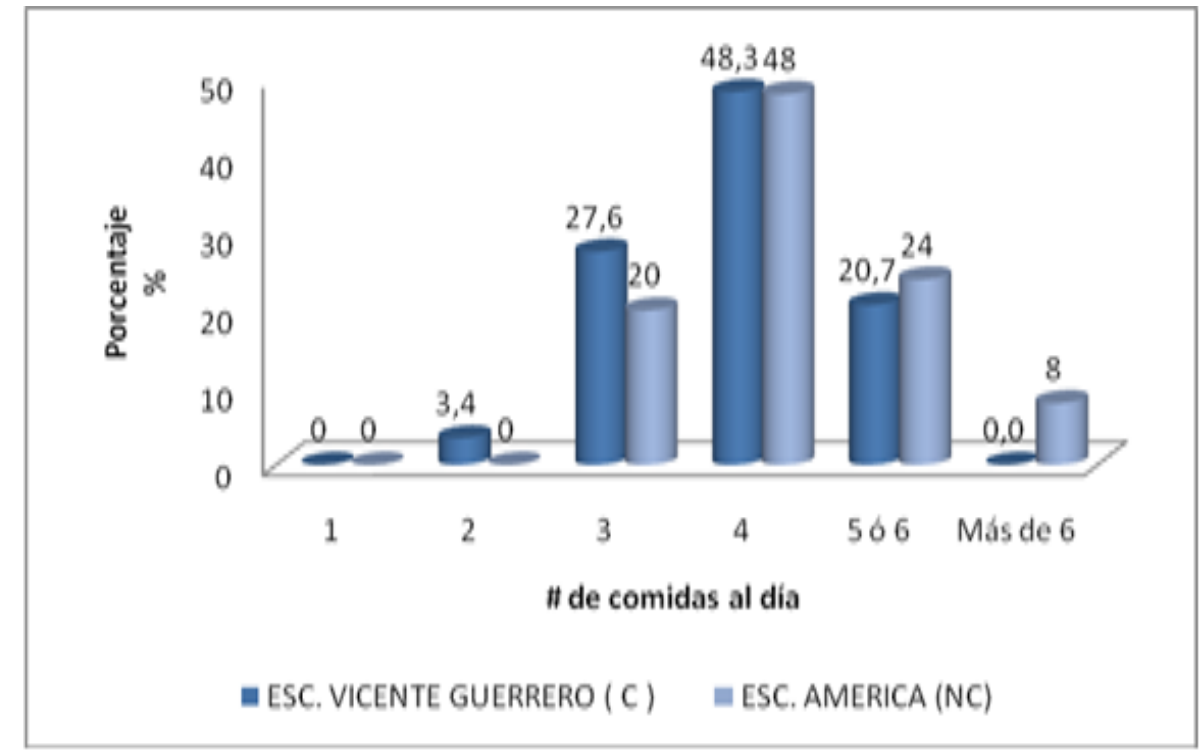

Fuente: elaboración propia.

En cuanto al número de niños que acostumbran desayunar antes de ir a su escuela el $51.7 \%$ perteneciente a la escuela $\mathrm{C}$ siempre desayuna, mientras que el $56 \%$ de la escuela $\mathrm{NC}$ a veces desayuna (Ver Gráfica No. 6). 
Gráfica No. 6. Alumnos que desayunan antes de ir a la escuela.

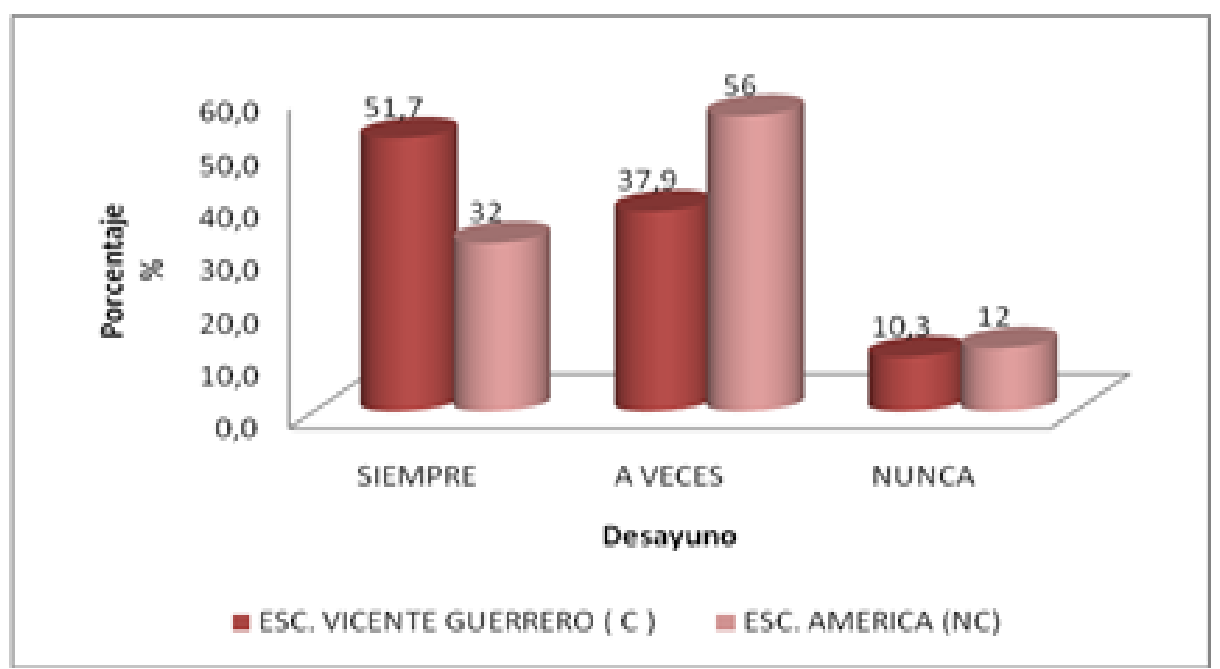

Fuente: elaboración propia.

Se preguntó a los niños cuál era el alimento que consumían diariamente, a lo que, los de la escuela $\mathrm{C}$, contestaron con mayor porcentaje que ninguno de los mencionados, siendo el refresco el alimento que más consumen (13.3\%) respecto al resto de los alimentos mencionados; situación semejante que refirieron los alumnos de la escuela NC, optando por el refresco en un $20 \%$ y en un $37.1 \%$ ninguno (Ver Gráfica No.7).

Gráfica No. 7. Alimentos consumidos diariamente por los alumnos.

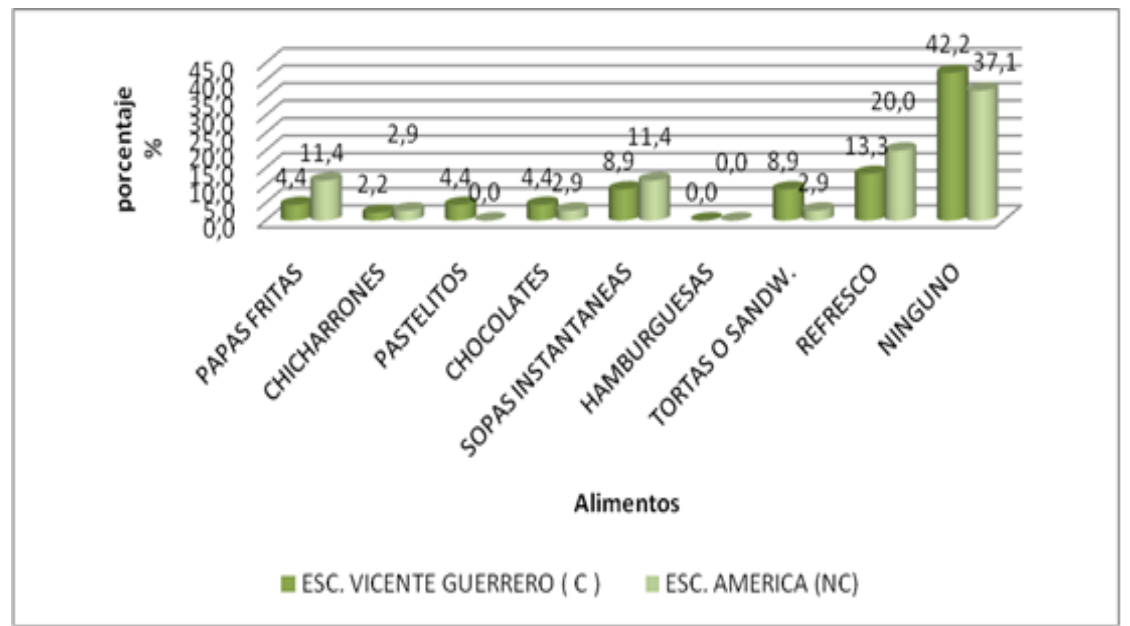

Fuente: elaboración propia. 
Así mismo se les preguntó cuántas veces al día consumen frutas y verduras. Según la Gráfica No.8, esto fue lo reportado: en ambas escuelas, suelen consumirlas de una a dos veces al día con un porcentaje de $62.1 \%$ y $56 \%$, respectivamente.

\section{Gráfica No. 8. Consumo al día de frutas y vegetales.}

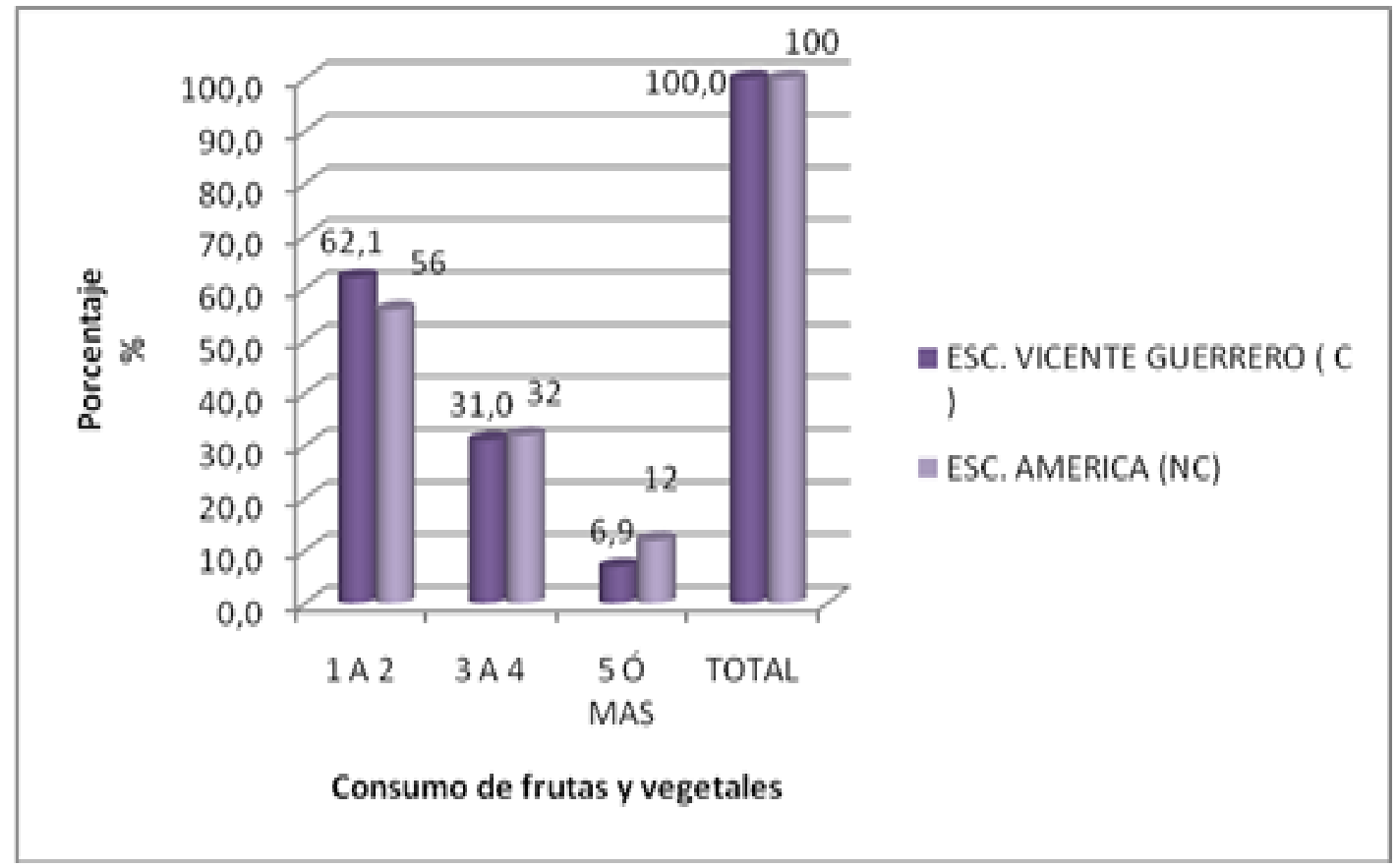

Fuente: elaboración propia.

En la Gráfica No. 9 se muestra la frecuencia en la que los escolares participantes realizan actividades físicas en su escuela, y en ambas la mayoría de los niños refirió practicarlas cinco veces por semana, sin embargo, en cuanto a los niños que no acostumbran a realizarlas, el mayor porcentaje (8\%) en comparación a la otra escuela (3.4\%) se presentó en la Escuela Primaria América. 
Gráfica No. 9. Número de veces a la semana que realizan actividades físicas en la escuela.

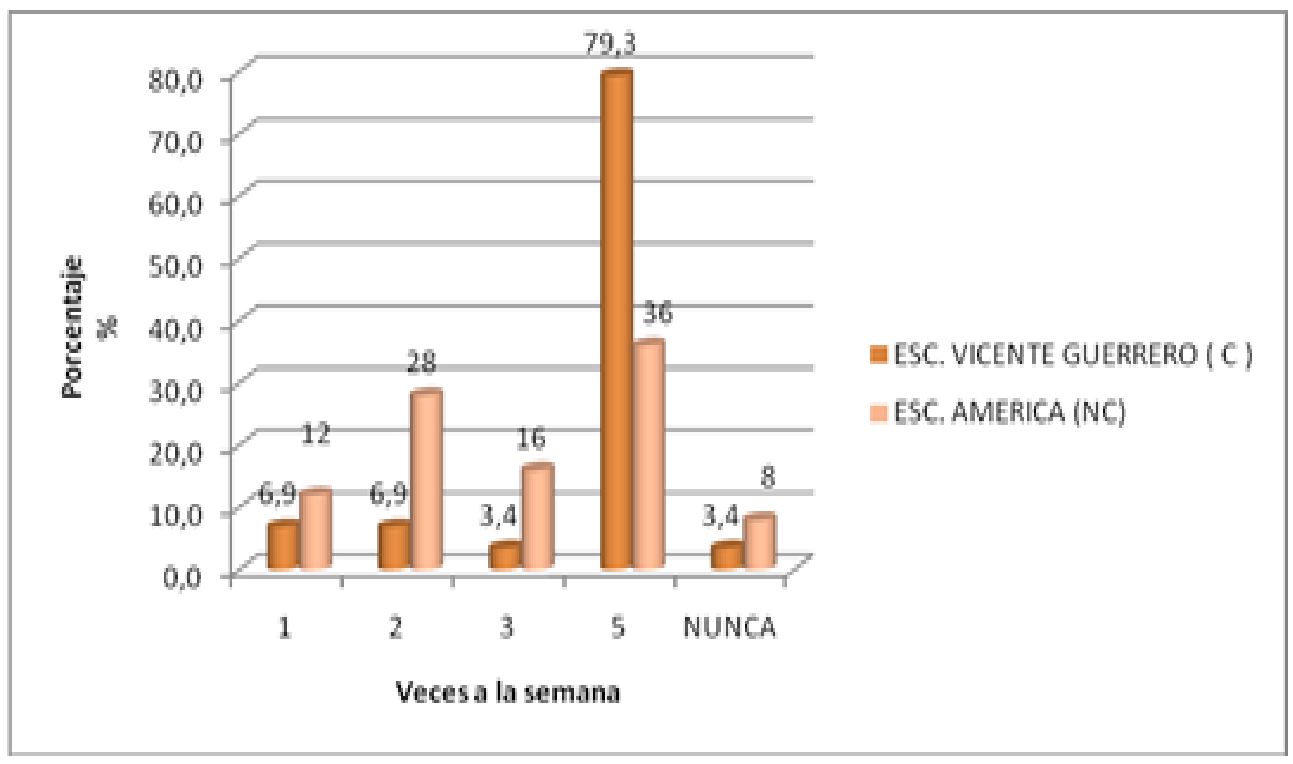

Fuente: elaboración propia.

La tabla No.7 se muestra las frecuencia con que los escolares de cada una de las escuelas juega video juegos, siendo los niños de la escuela $\mathrm{C}$ los que respondieron que lo hacen a diario con un $24.1 \%$, comparado con los alumnos de la escuela NC que lo hace en un ocho por ciento (8\%).

TABLA No. 7. Frecuencia de uso de video juegos.

\begin{tabular}{|c|c|c|c|c|c|}
\hline \multicolumn{6}{|c|}{ CADA CUANDO JUEGAN VIDEO JUEGOS } \\
\hline & DIARIO & A & FIN & $\mathrm{DE}$ & $\overline{\text { NUNCA }}$ \\
\hline & & VECES & SEMANA & & \\
\hline $\begin{array}{l}\text { ESC. VICENTE } \\
\text { GUERRERO (C) }\end{array}$ & 7 & 11 & 4 & & 7 \\
\hline$\%$ & 24.1 & 38 & 13.8 & & 24.1 \\
\hline $\begin{array}{l}\text { ESC. AMÉRICA } \\
\text { (NC) }\end{array}$ & 2 & 11 & 8 & & 4 \\
\hline$\%$ & 8.0 & 44.0 & 32.0 & & 16.0 \\
\hline
\end{tabular}

Fuente: elaboración propia. 
Menú ofrecido en la Escuela Primaria Vicente Guerrero

Ejemplo de menú de una semana:

TABLA No. 8. Menú de una semana ofrecido en la Escuela Primaria Vicente Guerrero.

\begin{tabular}{|c|c|c|c|c|}
\hline & LUNES & MARTES & MIÉRCOLES & JUEVES \\
\hline $\begin{array}{l}\text { PLATO } \\
\text { PRINCIPAL }\end{array}$ & $\begin{array}{l}\text { Guisado de } \\
\text { molida de res } \\
\text { con espinacas }\end{array}$ & Tortas de atún & Pollo empanizado & Caldo de res \\
\hline SOPA & Arroz rojo & Caldosa & Espagueti con queso & Arroz rojo \\
\hline ENSALADA & & & lechuga con tomate & \\
\hline POSTRE & $\begin{array}{l}\text { Galletas } \\
\text { canelitas }\end{array}$ & Gelatina de uva & Piña en almíbar & Naranja \\
\hline AGUA & Naranja & Jamaica & Natural & Limón \\
\hline $\begin{array}{l}\text { TOTAL } \\
\text { KCAL } \\
\text { \%VCT } \\
\text { HDC \% } \\
\text { PROT\% } \\
\text { LIP\% }\end{array}$ & $\begin{array}{l}709.5 \\
35 \\
72 \\
11 \\
17\end{array}$ & $\begin{array}{l}920 \\
46 \\
66 \\
11 \\
23\end{array}$ & $\begin{array}{l}810 \\
40.5 \\
42 \\
16 \\
42\end{array}$ & $\begin{array}{l}480 \\
24 \\
52 \\
17 \\
31\end{array}$ \\
\hline
\end{tabular}

Fuente: elaboración propia.

Datos obtenidos al analizar de los menús:

La escuela cuenta con Licenciado en Nutrición, quien es responsable de elaborar los menús y modo de preparación de los alimentos. - Alimentos preparados por madres de familia. - A los alumnos de $1^{\circ}, 2^{\circ}$ y $3^{\circ}$ se les proporciona el desayuno y a los de $4^{\circ}, 5^{\circ}$ y $6^{\circ}$ la comida. Siendo el mismo menú para todos. - Se utilizan tazas y cucharas medidoras, sin embargo la 
cantidad que sirven a los niños es al "tanteo". Sirviéndoles mayor cantidad a los grandes y poco a los chicos. - Se analizaron menús de dos semanas (cuatro días cada uno), encontrándose: Mínimo de calorías en menús: 469 Kcal, máximo de calorías en menús: 920 Kcal, promedio de: $630 \mathrm{Kcal}$ (ideal $600 \mathrm{Kcal}$ de dieta de $2000 \mathrm{Kcal}$ ), equivalente al 31.5\% de una dieta de $2000 \mathrm{Kcal}$, en promedio se estima: el 55\% son hidratos de carbono, el 16\% proteínas y el 29\% lípidos. - De los 8 menús analizados, sólo el 12.5\% fueron balanceados, mientras el $87.5 \%$ no lo fue. - Alto contenido de carbohidratos simples. - Bebidas de sabor en polvo. - Fruta en almíbar. - Galletas y panes con alto contenido de azúcar. - Alto contenido de grasa, especialmente de tipo polinsaturada y saturada. - Escasas frutas y verduras, y no se incluyen diariamente en menús. - El consumo de carnes blancas es bueno, de dos a tres días a la semana.

En ambas escuelas se cuenta con una "tiendita", a la cual los niños tienen libre acceso, sin embargo el tipo de productos alimenticios que se les ofrecen a los niños en cada una son distintos. En la escuela C, se aplica la "ley antichatarra", puesto que no se venden papas fritas ni chicharrones, y en su lugar se ofrecen palomitas sin mantequilla; no se venden refrescos ni jugos con alta cantidad de azúcar; tampoco se expenden golosinas con chile o tamarindo. Sólo se ofrecen alimentos preparados como sándwiches de jamón con pan integral y coctel de frutas. A diferencia de la escuela NC, donde aún no entra en rigor la "ley antichatarra" y se ofrece a los niños variedad de frituras con salsa, dulces, jugos, refrescos, donas, y alimentos preparados como hamburguesas, pizzas y burros.

\section{Discusión y conclusión}

De acuerdo a los resultados obtenidos en el presente estudio, se concluye lo siguiente:

Los casos de malnutrición se presentaron entre los nueve y 10 años edad. Siendo los escolares de 10 años de la escuela sin comedor los que presentaron sobre peso, a diferencia de la escuela con comedor, que no reportó ningún caso, sin embargo tuvo mayor número de casos de 
escolares en riesgo de sobre peso ente los nueve años y emaciación en los de 10 años.

El refresco es el alimento que diariamente consumen los niños de ambas escuelas, siendo más notorio en el plantel sin comedor. Al preguntar sobre el consumo de vegetales, el mayor porcentaje de los niños que respondieron consumirlos diariamente fue en la escuela $\mathrm{C}$, sin embargo el mayor porcentaje en el consumo de frutas a diario, fue en la escuela NC. En cuanto a la actividad física realizada dentro y fuera del plantel escolar, el mayor porcentaje de niños que la realizan con más frecuencia fueron los de la escuela $\mathrm{C}$, sin embargo son los que tienden a pasar más tiempo frente al televisor y jugando video juegos que los de la escuela NC.

El estado nutricional de los escolares de la escuela primaria con comedor (Vicente Guerrero) es diferente en comparación con los escolares de la escuela primaria sin comedor (América), sin embargo en ambos planteles educativos se observan casos de malnutrición, por lo que no se puede afirmar que el contar o no con el servicio de comedor escolar tuviera influencia en el desarrollo de problemas nutricionales. Sólo puede apreciarse que los escolares con comedor en su plantel educativo y con la "ley antichatarra" aplicada en su "tiendita" son los que mejores hábitos alimenticios refieren, lo cual es indispensable para gozar de una buena salud tanto en el presente de nuestra vida como en futuro.

\section{Recomendaciones}

Se recomienda hacer un estudio igual a éste utilizando una muestra de mayor magnitud, para obtener datos más significativos de problemas de malnutrición en escolares con servicio de comedor y escolares sin comedor en sus planteles educativos, y así establecer una comparación entre ellos. Además, se recomienda hacer un estudio longitudinal que evalúe el estado nutricional de escolares con comedor al inicio de la implementación de este servicio 
en el plantel y seis o 12 meses después, para observar el impacto del comedor escolar. 


\section{Referencias}

Aranceta, B.; Pérez, R.; Dalmau, J.; Gil, A.; Lama, R.; Martín, M.; Martínez, V; Pavón, P.; Suárez, L. "El comedor Escolar: situación actual y guía de recomendaciones". Asociación Española de Pediatría. (2008). 69(1), 72-88.

Arnau, J. "Comedores escolares y la salud de los niños". (2008). [En línea]. En BuenasManos.http://www.enbuenasmanos.com/articulos/muestra.asp? art=1868.

Brown, J. "Nutrición en las diferentes etapas de la vida”. Segunda edición. Editorial Mc Graw - Hill Interamericana Editores. México, D.F. (2006). 282, 286 y 287 pp.

Casanueva, E.; Kaufer, M.; Pérez, A.; Arroyo, P. “Nutriología Médica”. $3^{\circ}$ edición. Editorial Médica Panamericana. México. (2008).79, 108 y 109, 265 - 268, 278 y 279 pp.

Comedores Industriales. "Aplicación de los lineamientos generales para el expendio y distribución de alimentos”. Editorial Nuevo Norte. (2011). [En línea]. México. http://www.comedoresindustriales.com.mx/index.php?option=com_content\&view= article\&id=217: aplicacion-de-los-lineamientos-generales-para-el-expendio-ydistribucion-de-alimentos-y-bebidas-en-las-escuelas-de-educacion-basica-en-elpais\&catid=35:nutricion-y-salud\&Itemid=55.

ENSANUT. "Encuesta Nacional de Salud 2006”. (2006) [En línea]. México. http://www.facmed.unam.mx/deptos/salud/censenanza/spi/unidad2/ensanut2006.pdf

ENSANUT. "Encuesta Nacional de Salud 2012”. (2012). http://ensanut.insp.mx/doctos/ENSANUT2012_Nutricion.pdf

García, M. "Iniciativa con proyecto de decreto por el que se reforma el artículo $7^{\circ}$ de la Ley General de Educación”. Senado de la República LXI Legislatura, Servicios Parlamentarios, Diario de los Debates. (2010). [En línea]. México. http://www.senado.gob.mx/index.php?ver=sp\&mn=3\&sm=3\&lg=LXI_I\&id=1044

López, E.; Grijalva, M.; Valencia, M.; Ponce, J.; Artalejo, E. 'Impacto de un programa de desayunos escolares en la prevalencia de obesidad y factores de riesgo cardiovascular en niños sonorenses”. Salud Pública de México. (2005).47:126-133.

OMS (Organización Mundial de la Salud). ¿Qué son la obesidad y el sobrepeso? 1ra Edición. (2006). [En línea] U.S.: El comité. http://www.who.int/mediacentre/factsheets/fs311/es/index.html

OMS (Organización Mundial de la Salud). "Curso de capacitación sobre la evaluación del crecimiento del niño”. (2008). [En línea]. Ginebra: http://www.who.int/childgrowth/training/c_interpretando.pdf

Payán, A. "Desnutrición y obesidad son los retos”. Síntesis Razones de Ser, (2006). pp. Nacionales. 
Rodríguez, R. "Caducó el programa de desayunos escolares". [En línea]. México, El Universal. (2008). http://www2.eluniversal.com.mx/pls/impreso/version_imprimir.html?id_nota=1595 $35 \&$ tabla $=$ nacion. 